\title{
THE INTERSECTION MULTIPLICITY OF COMPACT $n$-DIMENSIONAL METRIC SPACES
}

\author{
GLENN P. WELLER
}

\begin{abstract}
It is shown that there is an integer $\mu(n)$ such that any compact $n$-dimensional metric space $M$ has intersection multiplicity at most $\mu(n)$. That is, if $\mathscr{U}$ is an open cover of $M$, then there is an open cover $\mathscr{V}$ refining $\mathscr{U}$ such that any element of $\mathscr{V}$ can intersect at most $\mu(n)$ other elements of $\mathscr{V}$.
\end{abstract}

For any open cover $\mathscr{U}$ of a topological space $X$ define $m(\mathscr{U})$ to be the maximum number of elements of $\mathscr{U}$ that any member of $\mathscr{U}$ can intersect. $X$ is said to have intersection multiplicity at most $m$ if and only if every open cover $\mathscr{U}$ of $X$ has an open refinement $\mathscr{V}$ covering $X$ such that $m(\mathscr{V}) \leqq$ $m$. The intersection multiplicity of $X$ is then the least integer $m$ such that $X$ has intersection multiplicity at most $m$ and is denoted $m(X)$.

THEOREM. For every integer $n$ there is an integer $\mu(n)$ such that, for every compact $n$-dimensional metric space $M, m(M) \leqq \mu(n)$.

This result is known [3, p. 301] for differentiable manifolds and follows from two lemmas, the first of which is quite clear.

LEMMA 1. If $A$ is a closed subset of $X$, then $m(A) \leqq m(X)$.

Lemma 2. If $I^{p}$ is the unit cube in Euclidean space $R^{p}$, then $m\left(I^{p}\right) \leqq 3^{p}$.

Proof. Consider the cell complex $J$ consisting of all cubes in $R^{p}$ whose vertices have integral coordinates and whose edges have unit length. Let $K$ be a simplicial subdivision of $J$ with no new vertices [1, p. 11]. Let $\mathscr{V}$ be the cover of $R^{p}$ consisting of the open stars of vertices in $K$. Let $v$ and $v^{\prime}$ be vertices of $K$ such that their open stars intersect. Then $v$ and $v^{\prime}$ are vertices of a simplex in $K$, and their coordinates can differ by at most the integer 1 . If $v$ is fixed, there are at most $3^{p}$ choices for $v^{\prime}$. Hence, $m(\mathscr{V}) \leqq 3^{p}$.

Now let $\mathscr{U}$ be an open cover of $I^{p}$. Then for a sufficiently large integer $q$ the linear transformation $L(x)=x / q$, when restricted to a subcomplex of $K$, gives a triangulation of $I^{p}$ fine enough so that the image under $L$ of $\mathscr{V}$, when restricted to $I^{p}$, refines $\mathscr{U}$. Thus $m\left(I^{p}\right) \leqq 3^{p}$.

Received by the editors April 21, 1970 and, in revised form, March 3, 1972. AMS 1970 subject classifications. Primary 54E35, 57A15, 54D20, 54F45, 55C10.

Key words and phrases. Metric space, open cover, intersection multiplicity. 
Proof of the Theorem. We may embed $M$ as a closed subset of $I^{2 n+1}\left[2\right.$, p. 148]. Set $\mu(n)=m\left(I^{2 n+1}\right)$.

\section{REFERENCES}

1. J. F. P. Hudson, Piecewise linear topology, Benjamin, New York, 1969. MR 40 \#2094.

2. J. G. Hocking and G. S. Young, Topology, Addison-Wesley, Reading, Mass., 1961. MR 23 \#A2857.

3. Y. Shikata, On the smoothing problem and the size of a topological manifold, Osaka J. Math. 3 (1966), 293-301. MR 35 \#6148.

Department of Mathematics, University of Illinois at Chicago Circle, ChICAGo, ILLINOIS 60680 